\title{
Highlights from PHENIX at RHIC
}

\author{
Rachid Nouicer ${ }^{1,2, *}$, for the PHENIX Collaboration \\ ${ }^{1}$ Physics Department, Brookhaven National Laboratory, Upton, New York 11973, United States \\ ${ }^{2}$ RIKEN-BNL Research Center, Brookhaven National Laboratory, Upton, New York 11973, United States
}

\begin{abstract}
Hadrons conveying strange quarks or heavy quarks are essential probes of the hot and dense medium created in relativistic heavy-ion collisions. With hidden strangeness, $\phi$ meson production and its transport in the nuclear medium have attracted high interest since its discovery. Heavy quark-antiquark pairs, like charmonium and bottomonium mesons, are mainly produced in initial hard scattering processes of partons. While some of the produced pairs form bound quarkonia, the vast majority hadronize into particles carrying open heavy flavor. In this context, the PHENIX collaboration carries out a comprehensive physics program which studies the $\phi$ meson production, and heavy flavor production in relativistic heavy-ion collisions at RHIC. In recent years, the PHENIX experiment upgraded the detector in installing silicon vertex tracker (VTX) at mid-rapidity region and forward silicon vertex tracker (FVTX) at the forward rapidity region. With these new upgrades, the experiment has collected large data samples, and enhanced the capability of heavy flavor measurements via precision tracking. This paper summarizes the latest PHENIX results concerning $\phi$ meson, open and closed charm and beauty heavy quark production in relativistic heavy-ion collisions. These results are presented as a function of rapidity, energy and system size, and their interpretation with respect to the current theoretical understanding.
\end{abstract}

\section{Physics Motivation}

Until now, Quantum Chromodynamics (QCD) is considered the fundamental theory of the strong interaction between quarks and gluons. Conforming to QCD, at ordinary temperatures or densities this force confines the quarks into composite hadrons. However, when the temperature reaches the QCD energy scale or its density rises to the point where the average inter-quark separation is less than $1 \mathrm{fm}$, hadronic matter under extremely dense and hot conditions undergoes a phase transition to form a Quark-Gluon Plasma (QGP or QCD matter) in which quarks and gluons no longer are confined to the size of a hadron $[1,2]$. Since the discovery of the QCD matter at the Relativistic Heavy Ion Colllider (RHIC) [3-7] at Brookhaven National Laboratory (BNL), the perception of the properties of strongly interacting matter at high temperatures has been a central goal of our research. This task is carried out by making measurements of multiple observables over a large range of energies and collision systems. To achieve this goal, RHIC provided collisions of gold-gold $(\mathrm{Au}+\mathrm{Au})$, copper-copper $(\mathrm{Cu}+\mathrm{Cu})$, uranium-uranium $(\mathrm{U}+\mathrm{U})$, copper-gold $(\mathrm{Cu}+\mathrm{Au})$, deuteron-gold $(d+\mathrm{Au})$, helium-gold $\left({ }^{3} \mathrm{He}+\mathrm{Au}\right)$, proton-gold $(p+\mathrm{Au})$ and proton-Aluminum $(p+\mathrm{Al})$ at center of mass energies from $\sqrt{s_{N N}}=7.7$ to

\footnotetext{
^e-mail: rachid.nouicer@bnl.gov
} 


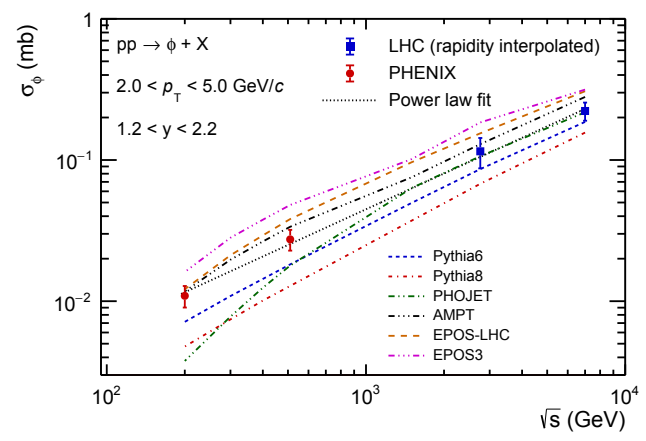

Figure 1. Production cross section of $\phi$ meson in $1.2<y<2.2$ and $2<p_{\mathrm{T}}<5 \mathrm{GeV} / c$ in $p+p$ collisions as a function of the center-of-mass energy $\sqrt{s}$. The experimental results are compared to different model predictions. The LHC data points are interpolated at the PHENIX forward rapidity, for details see Ref. [8]

$200 \mathrm{GeV}$, and proton-proton $(p+p)$ from $\sqrt{s}=62.4$ to $510 \mathrm{GeV}$ [7]. To study the properties of QCD matter created at RHIC, the PHENIX experiment established a comprehensive physics program which studies hadrons carrying strange quarks, open heavy flavor and quarkonium production as a function of rapidity, energy and system size.

\section{$2 \phi$ Meson Results}

The $\phi$ vector meson (the lightest bound state of $s$ and $\bar{s}$ quarks) is proposed as a good probe to investigate the properties of the QCD matter formed in heavy-ion collisions. A hydrodynamical study of transverse momentum $\left(p_{\mathrm{T}}\right)$ distribution of $\phi$ meson implies that it freezes out early compared to other hadrons $[9,10]$. The $\phi$ meson life time is about $42 \mathrm{fm} / \mathrm{c}$. Due to its relatively longer life time; the $\phi$ meson will mostly decay outside the fireball. Consequently, its daughter will not have much time to rescatter in the hadronic phase. This implies that properties of $\phi$ meson are basically controlled by the conditions in the early partonic phase which can be conceded as a good probe to investigate the properties of the QCD matter. Therefore, it is important to study the $\phi$ meson production as a function of centrality, rapidity, energy, and system size in heavy-ion collisions. Figure 1 shows the energy dependence of the $\phi$ meson production cross section integrated in $1.2<y<2.2$ and $2<p_{\mathrm{T}}<5 \mathrm{GeV} / c$ in $p+p$ collisions [8]. We observe that the experimental measurements of the $\phi$ meson production increase from RHIC to LHC energies and it follow a power-law versus the colliding energy defined as $\sigma_{\phi}(s) \propto s^{n}$, with $n=0.42 \pm 0.03$ (black dotted line). The results are compared to different model predictions, PYTHIA6, PYTHIA8, PHOJET, AMPT, EPOs3 and EPOS-LHC, for details see Ref. [8]. These models exhibit a similar energy dependence trend to that of the data. To study system size dependence of the $\phi$ meson production, the PHENIX experiment collected data from ${ }^{3} \mathrm{He}+\mathrm{Au}$ collisions in 2014 , and $p+\mathrm{Au}$ and $p+\mathrm{Al}$ collisions in 2015 RHIC runs. Figure 2 presents nuclear modification factors for minimum-bias ${ }^{3} \mathrm{He}+\mathrm{Au}, p+\mathrm{Au}$ and $p+\mathrm{Al}$ collisions at $\sqrt{s_{N N}}=200 \mathrm{GeV}$ as a function of transverse momentum (panels (a), (b) and (c)) and rapidity (panels (d), (e) and (f)). These results may provide a constraint on various cold nuclear matter effects included in models. 

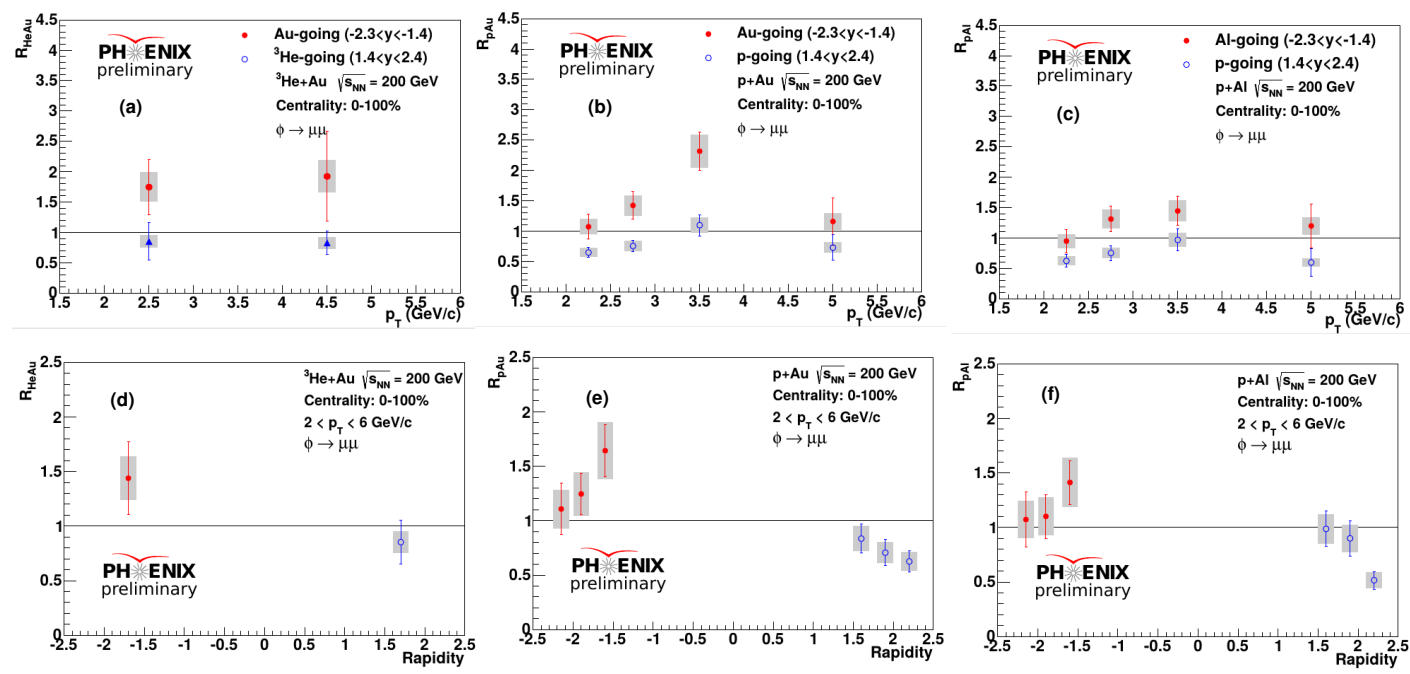

Figure 2. $\phi$ meson nuclear modification factors in minimum-bias ${ }^{3} \mathrm{He}+\mathrm{Au}, p+\mathrm{Au}$ and $p+\mathrm{Al}$ collisions at $\sqrt{s_{N N}}=200 \mathrm{GeV}$. Panels (a), (b) and (c), $\phi$ meson nuclear modification factors versus transverse momentum, $p_{\mathrm{T}}$. Panels (d), (e) and (f), $\phi$ meson nuclear modification factors versus rapidity.

\section{Heavy Flavor Results}

The PHENIX experiment studies heavy quark production via measurements of single leptons from semi-leptonic decays in both the electron channel at mid-rapidity and in the muon channel at forwardrapidity. To enhance the capability of heavy flavor measurements via precision tracking, the PHENIX collaboration has installed a silicon vertex tracker both in central rapidity (VTX) and in forward rapidity (FVTX) regions. The VTX [11] and FVTX [12] detectors cover respectively in rapidity $|y|<1.2$ and $1.2<y<2.2$, and $2 \pi$ in azimuth acceptance. With these two upgrades, the PHENIX experiment has collected large data samples. Recently, the PHENIX collaboration published first results on single electron yields from semileptonic charm and bottom decays in $\mathrm{Au}+\mathrm{Au}$ collisions at $\sqrt{s_{N N}}=200 \mathrm{GeV}$ using the VTX at mid-rapidity [13], and the FVTX published $B$-meson production at forward and backward rapidity in $p+p$ collisions at $\sqrt{s}=200 \mathrm{GeV}$ and $510 \mathrm{GeV}$, and $\mathrm{Cu}+\mathrm{Au}$ at $\sqrt{s_{N N}}=200 \mathrm{GeV}[15,21]$. These results will be discussed in the next sections.

\subsection{Separation of Bottom and Charm in Au+Au collisions at $\sqrt{s_{N N}}=200 \mathrm{GeV}$}

The large suppression $R_{\mathrm{AA}}$ of $e_{H F}^{ \pm}$at high- $p_{\mathrm{T}}$ observed in $\mathrm{Au}+\mathrm{Au}$ collisions at $\sqrt{s_{N N}}=200 \mathrm{GeV}$, and similar to that of light mesons $\pi^{0}$, is striking because perturbative QCD calculations indicate a substantial contribution from bottom quark decays for $p_{\mathrm{T}}>5 \mathrm{GeV} / \mathrm{c}$ [16]. For the specific purpose of separating the contributions of charm and bottom quarks at mid-rapidity, the PHENIX has added micro-vertexing capabilities in the form of a silicon vertex tracker (VTX). The different lifetimes and kinematics for $D$-meson $\left(c \tau_{D^{0}} \approx 123 \mu \mathrm{m}\right)$ and $B$-meson $\left(c \tau_{B^{0}} \approx 455 \mu \mathrm{m}\right)$ hadrons decaying to electrons enable separation of charm and bottom contributions with measurements of displaced tracks.

Figure 3 shows the $\mathrm{DCA}_{T}$ distribution (in transverse plane) for electrons $\left(e^{ \pm}\right)$in $2.0<p_{\mathrm{T}}^{e}<2.5 \mathrm{GeV} / \mathrm{c}$ range obtained in $0-10 \%$ central $\mathrm{Au}+\mathrm{Au}$ collisions at $\sqrt{s_{N N}}=200 \mathrm{GeV}$. In 

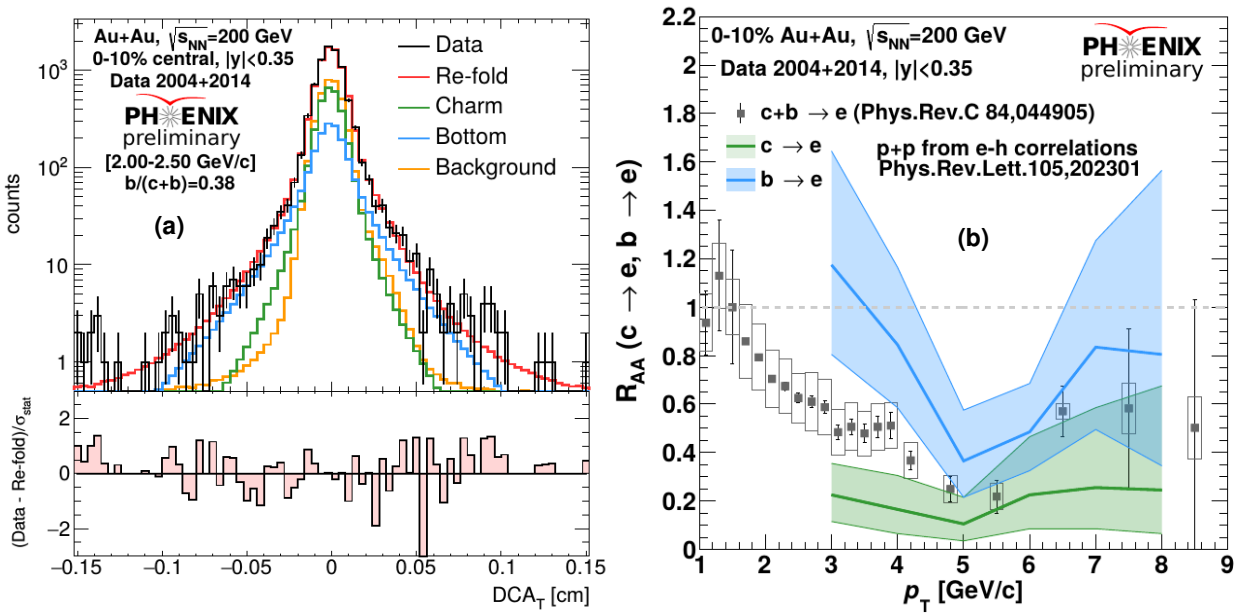

Figure 3. Panel (a) shows the $\mathrm{DCA}_{T}$ distribution for electrons with $2.0<p_{\mathrm{T}}^{e}<2.5 \mathrm{GeV} / \mathrm{c}$ in $\mathrm{Au}+\mathrm{Au} 0-10 \%$ central collisions at $\sqrt{s_{N N}}=200 \mathrm{GeV}$. The bottom and charm components from the unfolding method are also plotted. Also shown are the normalized contributions for the various background components. Panel (b) presents the nuclear modification factors $R_{\mathrm{AA}}$ of electrons from charm $(c \rightarrow e)$, bottom $(b \rightarrow e)$ decays and combined heavy flavor as a function of $p_{\mathrm{T}}^{e}$ in $\mathrm{Au}+\mathrm{Au} 0-10 \%$ central collisions at $\sqrt{s_{N N}}=200 \mathrm{GeV}$.

order to extract the heavy flavor contributions, all background components as shown in figure 3(a), were fully accounted for and their $\mathrm{DCA}_{\mathrm{T}}$ shapes as a function of $p_{\mathrm{T}}$ incorporated. Details of the method of subtracting all background components from $\mathrm{DCA}_{\mathrm{T}}$ shape can be found in Ref. [13].

The nuclear modifications, $R_{A A}^{c \rightarrow e}$, and $R_{A A}^{b \rightarrow e}$ gather in $0-10 \%$ central $\mathrm{Au}+\mathrm{Au}$ collisions at $\sqrt{s_{N N}}=200 \mathrm{GeV}$, for charm and bottom hadron decays respectively are presented in figure 3(c). We find that electrons from bottom hadron decays are less suppressed than those from charm for the region $p_{\mathrm{T}}<5 \mathrm{GeV} / \mathrm{c}$. We observe also that the result is consistent with the expectation of the mass ordering of energy loss. It should be point out that mass scale for the quark energy-loss may be relevant only for $p_{\mathrm{T}}<\mathrm{m}_{q}$, as figure 3(b) suggests. he work to finalize the results and provide more centrality classes are in progress.

\subsection{Measurement of $B$-meson $\rightarrow J / \psi$ in $p+p$ and $\mathrm{Cu}+\mathrm{Au}$ collisions}

The production of b-quarks are mainly from gluon fusion and flavor excitation at RHIC energy, and from split gluons at the LHC energy [17]. This characteristic may provide fundamental informations on how b-quarks lose energy in the QGP. While b-quarks from gluon splitting behave as a gluon when crossing the QGP plasma. However, b-quarks from fusion traverse the medium with a definite quantum number and their mass comes to be important for the amount of energy they lose in the medium [18]. Also plasma induced gluon splitting into b-quark pairs should be examined. At the LHC energy, the preeminence of gluon splitting may be the feasible reasons of why nuclear modifications of b-jets and inclusive jets are in good agreement $[19,20]$. To verify how initial state effects influence the $b$-quark production in heavy ion collisions is to measure $B$-meson yields integrated over momentum and centrality. One way to do this is to measure dimuon pairs produced from the $B$-mesons decay.

Datasets used in this analysis, measurements of the $B$-mesons $\rightarrow J / \psi$ s, including VTX and FVTX trackers in PHENIX experiment are from 2012 and 2015 RHIC runs. The 3D vertex position provided 

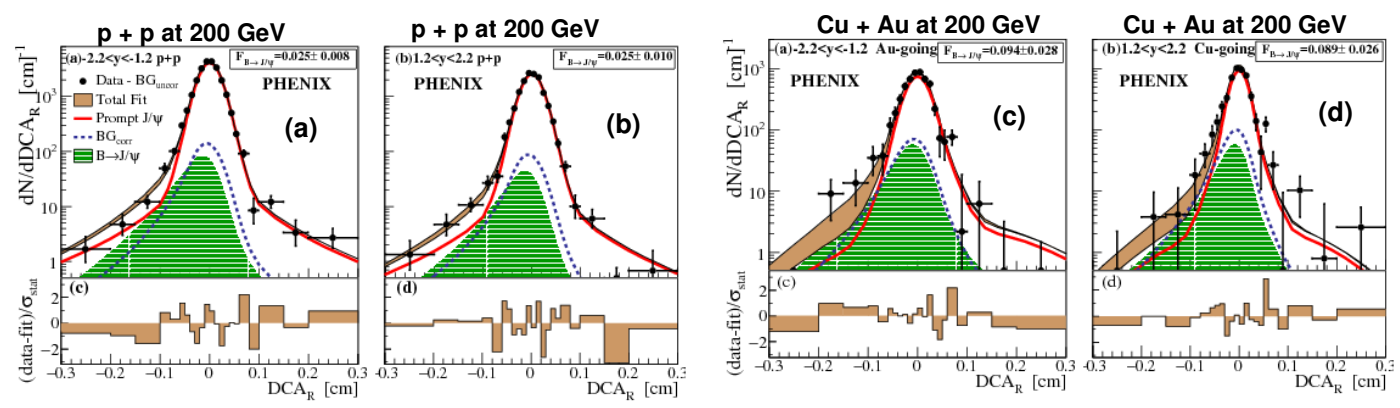

Figure 4. Radial distance of closest approach of single muons from dimuons in the $J / \psi$ mass region in $\mathrm{p}+\mathrm{p}$ and $\mathrm{Cu}+\mathrm{Au}$ collisions at $\sqrt{s_{N N}}=200 \mathrm{GeV}$. Combinatorial background and incorrect FVTX-MuTr associations are subtracted [15].
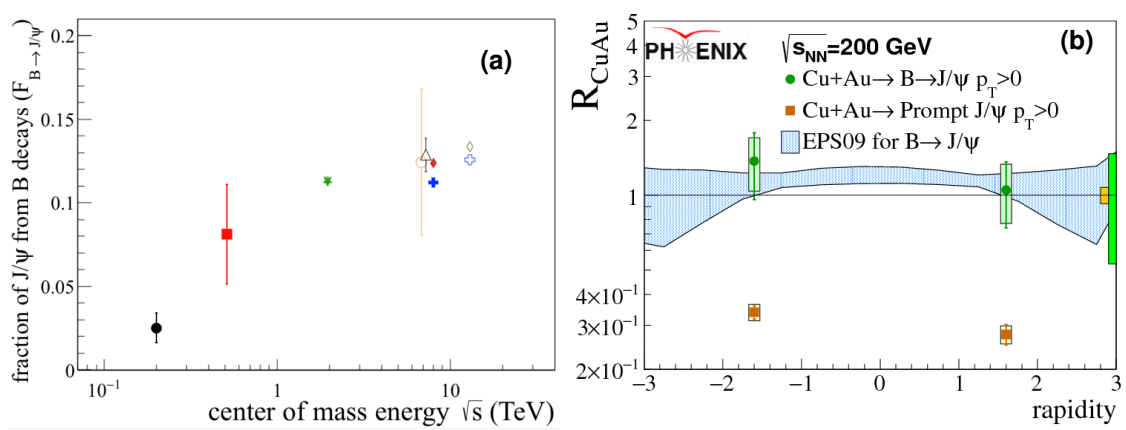

Figure 5. Panel (a) presents collision energy dependence of the fraction of $B$-meson $\rightarrow J / \psi$ [19]. Panel (b) shows nuclear modification factor of $B$-mesons and prompt $J / \psi$ in $\mathrm{Cu}+\mathrm{Au}$ collisions $[15,21]$ compared to an estimate from nuclear parton distribution modification (EPS09) [22].

by all VTX and FVTX tracks has a precision ranging from $200 \mu \mathrm{m}$ in $p+p$ collisions to $30 \mu \mathrm{m}$ in central $\mathrm{Cu}+\mathrm{Au}$ collisions. $J / \psi \rightarrow \mu^{+} \mu^{-}$decays are identified by the dimuon invariant mass peak in the muon arms, where each arm is composed of approximately one-meter thick hadron absorber, a muon tracker with three stations (MuTr) and a muon identifier (MuID). A MuTr+MuID identified $J / \psi$ can have one or both muons in the pair matching FVTX, all matchings are considered in the analysis. The muon track displacements are measured by the FVTX. The track displacement is defined by the radial projection of the distance of the closest approach (DCAR), in which direction the FVTX provides the best DCAR precision between 100-200 $\mu \mathrm{m}$. The DCAR distribution has contributions from the following sources: (a) dimuon combinatorial background; (b) incorrect matchings between the FVTX and the MuTr standalone tracks; (c) correlated dimuons in the $J / \psi$ mass region coming from $c \bar{c} \rightarrow \mu^{+} \mu^{-}$and $b \bar{b} \rightarrow \mu^{+} \mu^{-}$; and (d) prompt and non-prompt $J / \psi$. The fraction of non-prompt $J / \psi$ in the total $J / \psi$ yield $\left(\mathrm{F}_{B} \rightarrow J / \psi\right)$ is obtained from a likelihood fit of the DCAR distribution as shown in Fig 4. The fraction $\mathrm{F}_{B} \rightarrow J / \psi$ is the only free parameter in the fit. For more details about the analysis procedure see Refs. [15, 19,21]. Figure 5(a) shows measured $\mathrm{F}_{B} \rightarrow J / \psi$ in $p+p$ collisions at $\sqrt{s_{N N}}=200$ and $510 \mathrm{GeV}$ compared to the world data. The trend exhibits an increase from $\sqrt{s_{N N}}$ $=200 \mathrm{GeV}$ to $510 \mathrm{GeV}$ at RHIC and almost constant at Tevatron and LHC energies. Figure 5(b) 
presents the B-meson nuclear modification $\mathrm{R}_{\text {CuAu }}^{B-\text { meson }}$. It was obtained using $\mathrm{F}_{B} \rightarrow J / \psi$ results in $p+p$ and $\mathrm{Cu}+\mathrm{Au}$ collisions, and measured $J / \psi$ nuclear modification factor in $\mathrm{Cu}+\mathrm{Au}$ collisions $\left(\mathrm{R}_{C u A u}^{J / \psi}\right)$ all at the same energy, $\sqrt{s_{N N}}=200 \mathrm{GeV}$. The $\mathrm{R}_{C u A u}^{B-m e s o n}$ is in good agreement with binary scaling from $p+p$ collisions for $B$-mesons. Calculation of nuclear modification factor from initial state effects in EPS09 model [22] is in good accordance with experimental result. However, the $\mathrm{R}_{\text {CuAu }}^{B-m e s o n}$ for prompt $J / \psi$ exhibits a strong suppression, caused by breakup in the medium.

\subsection{Conclusions}

PHENIX measurements of hadrons conveying strange quarks or heavy quarks in heavy-ion collisions at RHIC have led to a broad and deep understanding of the properties of hot QCD matter. The PHENIX measurements of $\phi$ meson production in $p+p$ collisions imply an increase as a function of energy from RHIC to LHC, and it follows a power-law. For the first time at RHIC, measurements of charm and bottom separation are obtained in $0-10 \%$ central $\mathrm{Au}+\mathrm{Au}$ at $\sqrt{s_{N N}}=200 \mathrm{GeV}$ via single electrons from semi-leptonic $D$ and $B$ meson decays. We find that electrons from bottom hadron decays are less suppressed than those from charm for the region $p_{\mathrm{T}}<4 \mathrm{GeV} / \mathrm{c}$ and it is consistent with the expectation of the mass ordering of energy loss. We also presented the first results of $B$-meson $\rightarrow J / \psi$ in $p+p$ collisions at $200 \mathrm{GeV}$ and $510 \mathrm{GeV}$, and $\mathrm{Cu}+\mathrm{Au}$ at $200 \mathrm{GeV}$. As a function of energy, the trend $\mathrm{F}_{B} \rightarrow J / \psi$ measurements exhibits an increase from $\sqrt{s_{N N}}=200 \mathrm{GeV}$ to $510 \mathrm{GeV}$ at RHIC and almost constant at Tevatron and LHC energies.

\section{References}

[1] E. Shuryak, Phys. Rep. 61, 71 (1980).

[2] L. McLerran, Rev. Mod. Phys. 58, 1021 (1986).

[3] I. Arsene et al. (PHENIX Collaboration), Nucl. Phys. A 757, 1 (2005).

[4] B. B. Back et al. (PHOBOS Collaboration), Nucl. Phys. A 757, 28 (2005).

[5] J. Adams et al. (BRAHMS Collaboration), Nucl. Phys. A 757, 102 (2005).

[6] K. Adcox et al. (STAR Collaboration), Nucl. Phys. A 757, 184 (2005).

[7] R. Nouicer, European Physical Journal Plus 131, 70 (2016), and references therein.

[8] A. Adare et al. (PHENIX Collaboration), arXiv:1710.01656v1 (2017).

[9] J. Adams et al. (STAR Collaboration), Nucl. Phys. A 757, 102 (2005).

[10] M. Nasim et al., Adv. High Energy Phys. 2015, 197930 (2015).

[11] R. Nouicer et al., Nucl. Inst. and Meth. in Phys. Res. B 261, 10671071 (2007).

[12] C. Aidala et al., Nucl. Inst. and Meth. in Phys. Res. A 755, 44 (2014).

[13] A. Adare et al. (PHENIX Collaboration), Phys. Rev. C 93, 034904 (2016).

[14] C. Aidala et al. (PHENIX Collaboration), Phys. Rev. D 95, 092002 (2017).

[15] A. Adare et al. (PHENIX Collaboration), Phys. Rev. C 96, 064901 (2017)

[16] M. Cacciari et al., Phys. Rev. Lett. 95, 122001 (2005).

[17] E. Norrbin et al., Phys. Lett. B 442, 407 (1998).

[18] J. Huang et al., Phys. Lett. B 726, 251 (2013).

[19] C. L. da Silva et al. (PHENIX Collaboration), Nucl. Phys. A 967, 652 (2017).

[20] S. Chatrchyan et al., Phys. Rev. Lett. 113, 13 (2014) 132301.

[21] A. Adare et al. (PHENIX Collaboration), Phys. Rev. D 95, 092002 (2017).

[22] K. J. Eskola et al., JHEP 04, 065 (2009). 